\title{
Anatomycal and histological structure of aortic valve in Baikal seal
}

\author{
V.N. Tarasevich* \\ Irkutsk State Agrarian University named after A.A. Ezhevsky, 664038 Irkutsk, Russia
}

\begin{abstract}
This article studies peculiarities of the anatomical and histological structure of the aortic valve cusps in Baikal seal in the age of 3-3.5 years. Topographically, the aortic valve takes a central position and is located between the atrioventricular valves, and cranial - pulmonary valve. Projectively, the aortic valve is placed above the level of the atrioventricular valves, but below the level of the pulmonary valve. The aortic ostium has marked sinuses, two coronary and one septal, forming the aortic bulb. The valve is shown by the septal right and left semilunar cusps. The septal cusp is caudally attached to the aortic ostium and the right and left have a cranial location. Aortic valve cusps have three layers of connective tissue: fibrosa (aortic) - with collagen fibers, ventriculosa (ventricular) - with elastic and spongiosa (intermediate) - a combination of both fibers, give strength to this structure. The thickness of the ventricular layer of the cusp is 2.4 times higher than the thickness of the aortic layer. At the places of the greatest thickness value, there are transverse resections ( 2 deep and 4 surface), which increase the contact surface and increase the pressure on the cusp. The thinning area, which is located before the bulging edge of the cusp, acts as a closure point for the aortic valve cusps.
\end{abstract}

\section{Introduction}

There are two circles of blood circulation in the body of animals: through the small (pulmonary) circuit, oxygen enrichment is ensured, and through the large (systemic) one the delivery of nutrients and $\mathrm{O} 2$ to the tissues of the body and excretion. Sanguimotion in the circulation is done through a hollow muscular organ - heart, which, due to the presence of a valvular heart apparatus, helps to transmit the blood in one direction [6]. The valves control the blood stream, opening and closing during contractions and compliance of the heart [13].

Significant data concerning the morphology of the human heart and various animal species has been collected by V.N. Zhedenov (1954) [3]; D.V. Vasilyev, et al. (2014) [2]; A.E. Kuzin (2015) [7]; R.A. Zhilin, I.P. Korotkova $(2015,2020)$ [4, 5]; I.I. Silkina (2020) [9]; Ryadinskaya N., et al. [15] and others.

Meanwhile, the heart of the Baikal seal has a number of morphologically adapted features: two apexes, placed almost horizontally from the 3 rd to the cartilage of the 9 th rib.

\footnotetext{
* Corresponding author: tarasevich7239@mail.ru
} 
It has an explanted and spherical shape, as indicated by the heart index of 1.25 (width / length) and 2.48 (width/ thickness), and the value of the relative weight of the heart, in puppies, is at the level of $-0.88 \%$ [12]. The tricuspid valve has three cusps, seven papillary muscles (large and additional, four septum and one hyparterial, from 26 to 28 tendinous cords $[10,11]$.

Nevertheless, no data found on the anatomical and histological structure of the semilunar aortic valve in the Baikal seal aged 3-3.5 years, which was the aim of the study.

The aim of the study is to examine the anatomical and histological structure of the semilunar aortic valves in the Baikal seal.

\section{Materials and methods}

The material for the research was hearts from female Baikal seals aged 3-3.5 years. We used the following methods in our work: fine dissection, photographing, and histotechniques. Tweezers suggested for working with the lymphatic system were used for the convenience of collecting histological material [8]. The pieces of the valve structure were fixed in $10 \%$ neutral formalin. After that, using the wiring method, they were placed in paraffin blocks through isopropyl alcohol and mineral oil (Buesa R., Peshkov M. 2011) [1]. Histological sections with a thickness of 8-10 microns were stained using iron hematoxylin (van Gieson's stain). Morphometric dimensions were made by a Lomo Micmed-6 microscope, Levenhuk C510 NG digital camera and Toup View software (x64).

\section{Results and discussion}

Aortic ostium is placed between the aorta itself and the arterial cone of the left ventricle. It is located above the level of the projected location of the atrioventricular openings, but below the pulmonary trunk entrance. (Fig. 1)

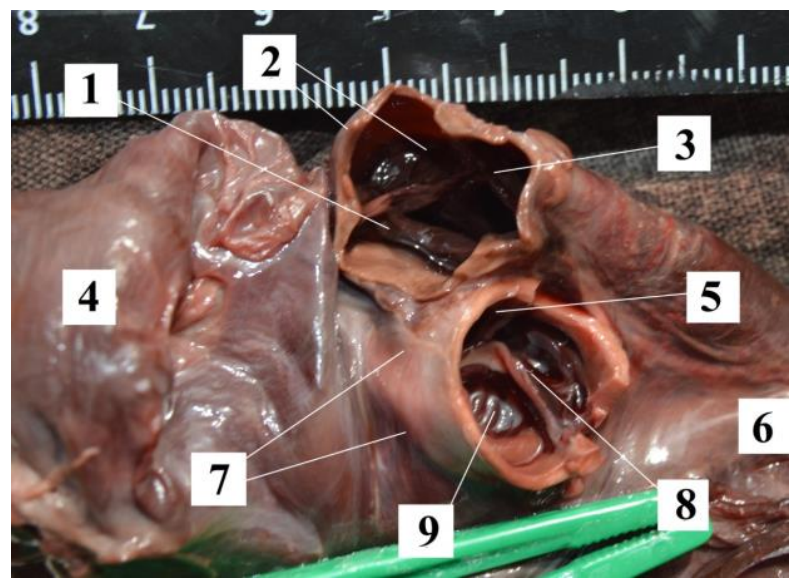

Fig. 1. Heart base of Baikal seal:

1 - left cusp of the semilunar valve of the pulmonary trunk; 2 - the entrance of the pulmonary trunk and the right semilunar cusp; 3 - intermediate semilunar cusp; 4 - left atrium; 5 - left semilunar aortic valve cusp; 6 - right atrium; 7 - aortic ostium; 8 - right semilunar aortic valve cusp; 9 - bottom part of the sinus of the septal cusp of the aortic valve.

Its lower part is bounded by a fibrous ring - the aortic hiatus; in the middle part - the sinuses of the ostium and the aortic valve; in the upper part-the sinotobular junction [10]. 
The fibrous ring is shaped like a crown with three teeth, which is formed by dense connective tissue. In the area of aortic hiatus, the fibrous ring has a height of $2 \mathrm{~mm}$ and an endocardial thickness of 456.6 microns. In the area of aortic ostium, the Baikal seal has well-defined sinuses, two coronary and one septal, forming the so-called aortic bulb. Whales and seals also have a well-marked aortic sinus [7].

The bottom part of the pockets (sinuses) of the aortic valve is placed above the myocardium of the base of the ventricles, which lowers the load on the valve cusps during the regurgitation. In this area, the thickness of the endocardium ranges from 273.7 to 295.5 microns.

The aortic valve in the Baikal seal is presented by the septal, right and left semilunar valves. The septal cusp is attached to the aortic ostium caudally, and the right and left have a cranial distribution. Nevertheless, according to A. A. Torshkov, they have the opposite distribution in cattle and pigs [13].

On tissue specimen of the lengthwise section of the aortic valve (Fig. 2) is visualized unevenness of the distribution of the cusp thickness. Its greatest value with a variation from 406 to 424.3 microns is noted almost throughout its entire length, with the exception of the zone of the cusp before its end, where the thickness for $1 \mathrm{~mm}$ is in the range of $173.5-$ 156.1 microns.

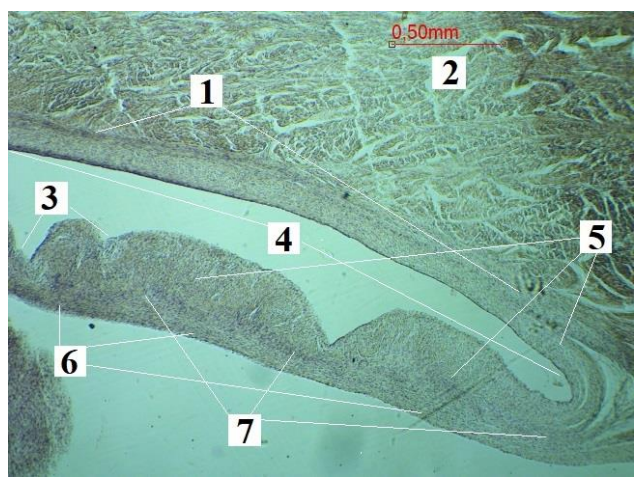

Fig. 2. Lengthwise section of the aortic valve cusp base, Baikal seal 3 years old ( $q$ ); van Gieson's stain, 256-fold zoom: 1 - outer coat of the endocardium; 2 - left ventricular myocardium; 3 - aortic valve cusp resections; 4 - bottom part of the aortic valve sinuses; 5 - aortic layer (fibrosa); 6 - inner ventricular layer (ventriculosa); 7 - spongiosa.

It is noteworthy that there are transverse resections. There are about six of them throughout the cusp length, two deep ones - up to 238.3 microns and 4 surface ones - from 46 to 78.4 microns, which presumably increase the contact area of the blood column with the cusp surface. The cusp edge on the longitudinal section has a thickening, rounded shape, and the thickness value in this part of the cusp was-242 microns.

The cusps forming the aortic valve are made up of three layers of connective tissue (aortic, ventricular, and spinous), covered outwardly by a thin endothelial layer. Layers adverted to the aortic lumen (fibrosa) (Fig. 2), mainly contain collagen fibers. The layer thickness at the base of the cusp is in the range of 113.5-147.4 microns. The middle layer (spongiosa) has a friable structure of fibrous tissue with a content of elastic and collagen fibers. In accordance with the data of a number of authors M. H. Yacoub et al. (1999), spongiosa is the backbone of each valve cusp and is made up of a dense collagen layer, which, in contrast to the ventricular layer, has a loose structure [14]. The inner ventricular layer is made up of a layer of dense connective tissue with elastic fibers - ventriculosa, which passes from the endocardium. Its thickness is insignificant and ranges from $47.3 \pm 1.13$ microns. 


\section{Conclusion}

1. From the topographical point of view, the aortic valve has a central location and is placed between the atrioventricular valves and the cranial valve of the pulmonary trunk. The aortic valve is projected above the level of the atrioventricular valves, but below the level of the pulmonary valve.

2. The aortic ostium has marked sinuses. They are: two coronary and one septal, forming the so-called aortic bulb. The valve is presented by the septal, right and left semilunar valves. The septal cusp is caudally attached to the aortic ostium and the right and left have a cranial distribution.

3. Aortic valve cusps have three layers of connective tissue: fibrosa (aortic) - with collagen fibers, ventriculosa (ventricular) - with elastic and spongiosa (intermediate) - a combination of both fibers, give strength to this structure. Ventricular cusp layer thickness is 2.4 times higher than the one of aortic layer. In the places of the greatest thickness value, the existence of transverse resections ( 2 deep and 4 surface) is noted, which enhance the contact area and expand the pressure on the cusps. The thinning area, situated in front of the thickening of the edge of the cusp is the place where cusps of the aortic valve are closed.

\section{References}

1. R.Kh. Buersa., Arkh. Patol, 73(1), 54 (2011)

2. D.V. Vasilyev, Ippol. i veterin, 4(14), 92 (2014)

3. V.N. Zhedenov, The lungs and heart of animals and humans, 202 (1954)

4. R.A. Zhilin, Vest. KrasGAU, 12, 220 (2015)

5. R.A. Zhilin, Vest. IrGSKhA, 98, 43 (2020).

6. N.V. Zelenevsky, Anatomy of dogs, 340 (1997)

7. A.E. Kuzin, Izv. TINRO, 182, 69 (2015)

8. Yu. M. Malofeev, Agrar. Vest. Ur. 6, 55 (2009)

9. I.I. Silkin, Morphological features of the reed warbler heart inhabiting in the Lake Baikal ecosystem, 304 (Ufa, 2020)

10. V.N. Tarasevich, Vest. IrGSKhA, 98, 111 (2020).

11. V.N. Tarasevich, N.I. Ryadinskaya, Morfol. 157(2-3), 208 (2020)

12. V.N. Tarasevich, Ippol. i veterin. 3(23), 178 (2020)

13. A.A. Torshkov, Izv. Orenburg. Gosud. Agrar. Univer. 1(9), 89 (2006)

14. M.H. Yacoub, P.J. Kilner, E.J. Birks, M. Misfeld, Ann Thorac Surg, 68(3), 37 (1999)

15. N. Ryadinskaya, Turk. J. of Zool, 44(1), 60 (2020) 